\title{
Supersonic Jet Noise from Round and Chevron Nozzles: Experimental Studies
}

\author{
R.H. Schlinker, J.C. Simonich, D.W. Shannon, R.A. Reba \\ United Technologies Research Center, E. Hartford, CT., 06108 \\ T. Colonius, K. Gudmundsson \\ Caltech, Pasadena, California, 91125, USA \\ F. Ladeinde \\ TTC Technologies, Inc., Stony Brook, New York, 11790, USA
}

\begin{abstract}
High speed exhaust noise reduction continues to be a research challenge for supersonic cruise business jets as well as for current and future tactical military aircraft. Significant noise reduction may be possible from advanced concepts for controlling instability generated large-scale turbulence structures in the jet shear layer, generally accepted to be the source of aft-angle noise. In response to this opportunity, our team is focused on experimental diagnostic studies and unique instability modeling suited for identifying control strategies to reduce large scale structure noise. The current paper benchmarks the jet noise from supersonic nozzles designed to provide the supporting experimental data and validation of the modeling. Laboratory scale jet noise experiments are presented for a Mach number of $\mathrm{Mj}=1.5$ with stagnation temperature ratios ranging from $T r=0.75$ to 2 . The baseline configuration is represented by a round converging-diverging (CD) ideal expansion nozzle. A round CD nozzle with chevrons is included as the first of several planned non-circular geometries directed at demonstrating the impact on large scale structure noise and validating noise prediction methods for geometries of future technological interest. Overexpanded and underexpanded conditions were tested on both nozzle configurations. The resulting data base provides an opportunity to benchmark the statistical characteristics of round and chevron nozzle data. The current paper examines far field spectra, directivity patterns, and overall sound pressure level dependence comparing observed characteristics with the fine scale turbulence noise and large-scale turbulence structure noise characteristics identified by Tam. In addition, the paper probes the effect of chevrons on the developing flow field and suppression of screech tones. Measurements are also reported from a far-field narrow aperture phased array system used to map the acoustic source distribution on the jet axis. The dominant source region, situated between the end of the potential core and the sonic point, was found to agree with the peak amplitude location of the jet near field wavepackets measured using a unique near field array. This observation supports the cause-effect link between large-scale turbulence structures in the shear layer and their dominant contribution to aft radiated far field noise.
\end{abstract}

\section{Introduction}

\begin{abstract}
A. Motivation
While subsonic jet noise has been dramatically reduced in the commercial aircraft engine sector largely through engine cycle changes and increasing bypass ratio, supersonic exhaust jet noise reduction continues to be a research challenge for supersonic cruise business jets currently under study within the industry as well as for current and future tactical military aircraft. Aircraft designed for supersonic cruise operation are typically seeking small diameters, low drag and weight, and high specific thrust engines resulting in very high exhaust velocities. Temperature augmentation via afterburning or outer stream duct burning has also
\end{abstract}


been considered within past optimization studies in order to minimize engine size and weight for supersonic commercial and business jets. Afterburner operation to increase thrust is used in current and future high performance military applications. Therefore, a better understanding of jet exhaust noise, its mechanisms, and propagation of high intensity noise sources is important to both the commercial and military arenas.

The topic is also motivated by the high noise levels experienced by ground crews and aircraft carrier launch/recovery crews as well as residential communities in close proximity to operational air bases. While the crew noise problem can be alleviated somewhat with personal protection equipment, the reduced noise intensity still exceeds desired levels by large margins, even for new protection gear in the research pipeline. Hence, jet noise control options are being researched on the engine side for personnel protection to be effective.

Current research programs directed at supersonic engine exhaust noise reduction are demonstrating benefits of 3-4 dBA using passive methods to increase jet mixing and break up shock cells in over-expanded flows. Approaches include mechanical chevrons, fluid/air injection used as deployable chevrons, and lobed nozzle inserts to achieve ideal expansion. Arguably, achieving dramatic improvements in noise reduction will require a multi-faceted approach combining unique engine/airframe architectures, and new componentlevel technologies.

\section{B. Long Term Goal and Relationship to Current Study}

In the long term, our research effort aims to develop component-level technologies based on controlling instability wave generated large-scale turbulence structures in the jet shear layer, generally accepted to be the source of aft-angle noise. Noise control strategies are envisioned based on modifying the instability wave spatio-temporal structure by introducing unsteady forcing in the initial shear layer region. Since this region is highly receptive to perturbations, the actuator authority and power requirements will be significantly lower compared to requirements for steady forcing reported in prior studies.

While this concept is not new, its development and impact on noise reduction has been hindered by the lack of physics-based forcing and control strategies. Therefore, a key objective of the current program is to develop fundamental knowledge and innovative modeling to identify such strategies. The team has focused on conducting research studies directed at experimental diagnostics and unique modeling capabilities suited for use in identifying control strategies to reduce large-scale structure noise in single stream supersonic exhaust flows.

The approach consists of multiple elements reported in different venues as described here briefly to anchor the current paper. First, the team is developing models to predict the near-field pressures associated with large-scale turbulent structures (wave packets). These models are based on representations of the pressure field as instability waves evolving in the turbulent mean flow field as summarized in References 1, 2 for the supersonic conditions reported here. In order to develop an integrated noise model, these formulations are based on RANS descriptions of the mean flow field and Parabolized Stability Equations (PSE) in addition to nonlinear PSE (NPSE) to account for the non-parallel and non-linear effects in the shear layer. Reference 3 develops a procedure for projecting the near field pressures, via solution of the wave equation, to the far-field, thus developing a quantitative cause-and-effect relationship between perturbations to the shear layer and far-field sound. This method has been extended to subsonic flows as reported in a companion conference paper under Reference 4.

A key enabler in this procedure is the development of near-field microphone arrays capable of providing the pressure statistics needed to validate the instability wave models or wavepackets. These methods detect the unsteady pressure signatures of organized turbulent structures at the interface between the jet flow and the acoustic radiation field. This region, located in the jet hydrodynamic near field, is viewed as the sound "source" containing the traveling wave pressure signature responsible for noise radiating to the far field in the aft direction. The source field can be measured with microphones just outside the non-linear turbulent 
flow region providing spectral features and length scales of the large-scale turbulence noise sources.

The first experimental diagnostic method linking near-field source measurements and far-field sound was presented by Reba et al. in Ref. 5. That study was limited to subsonic jets, and utilized near-field array data acquired by Suzuki and Colonius (Ref. 6) in partnership with NASA Glenn Research Center. Since then a second generation diagnostic array has been developed, built on the methods in Ref. 6, and first applied to an $\mathrm{Mj}=1.5$ jet in Reference 3. The approach minimizes the number of microphones with the latest application being for subsonic exhaust streams as reported in Reference 4. The subsonic and supersonic results show that the far field, reconstructed from the measured near field via analytical projection methods, agrees well with directly measured far field spectra. This demonstrates for the first time, that stability based methods may lead to predictive capability for analysis and design of nozzles and control schemes to mitigate organized structure noise.

While the studies in References 1-4 show progress towards developing shear layer instability models and near field to far field projection methods for predicting large-scale structure noise generated by round nozzles, the suppression of this mechanism will require alternative nozzle geometries and flow tailoring or excitation schemes. To validate the analysis methodology beyond round nozzles, a chevron nozzle has been designed and tested with the intent of applying the same instability formulation, near field diagnostics, and projection method to a geometry known to reduce far field noise. The recently acquired diagnostics will be compared with PSE predictions under development by Caltech to validate the prediction of organized structure modification and noise reduction via the chevron nozzle geometry. Comparisons with a baseline round nozzle will provide insight into the modified evolution of the shear layer instability. This work is still in progress.

\section{Current Study and Approach}

The current study is the first of several non-circular nozzle geometries planned to provide data bases for future assessment of nozzles of technological interest. Laboratory scale jet noise experiments are presented for a Mach number of $\mathrm{Mj}=1.5$ with stagnation temperature ratio ranging from $\operatorname{Tr}=0.75$ to 2 . The baseline configuration is represented by the round converging-diverging (CD) ideal expansion nozzle for which preliminary results were reported in References 3 and 7. A round CD nozzle with chevrons has now been added to evaluate the chevron impact on large scale structure noise for the validation. Off-design overexpanded and underexpanded flow conditions were investigated for both nozzles as the test program expanded from the initial study.

The resulting data base provides an opportunity to benchmark the statistical characteristics of round and chevron nozzle data. The current paper examines far field spectra, directivity patterns, and overall sound pressure level (OASPL) dependence on velocity. Observed characteristics are compared with the fine scale turbulence noise and organized structure noise mechanisms identified by Tam (References 8, 9). In addition, the paper probes the effect of chevrons on the flow field evolution and suppression of screech tones. Results are also reported for a new far- field narrow aperture phased array used to map the acoustic source image on the jet centerline. This result is compared with the organized structure noise generation region measured using a unique near field array.

\section{Experimental Approach}

\section{A. Facility and Jet Noise Rig}

Experimental studies were conducted in the United Technologies Research Center (UTRC) Acoustic Research Tunnel (ART) developed in 1970 as the first forward flight anechoic simulation facility for jet noise, fan and propeller noise, and lifting surface/airframe noise studies. A recent description is given by Simonich et al. in Reference [10]. The facility provides up to a 50" open jet forward flight simulation for jet noise using large single stream exhaust nozzles up to 6" diameter to achieve relevant Reynolds numbers, 
stagnation temperatures up to $1800 \mathrm{deg}$, and Mach numbers (M) up to 2.5 based on the 400 psi supply air.

The exhaust nozzles project beyond the open jet as shown in Figure 1. Boundary layer suction exists on the model nozzle exterior to control boundary layer thickness for simulation of full-scale engine nacelle external flows. The free field microphones located outside the open jet flows $(\mathrm{M}=0.1$ to 0.36$)$ provide sound pressure level measurements over the key jet noise directivity angles ranging from 80 deg to 155 deg from the engine inlet centerline. Higher Mach numbers up to $M=0.6$ are available with a 21 "x31" open jet test section.

The test section is surrounded by a sealed anechoic chamber 16 feet high, $18 \mathrm{ft}$ long (in the jet centerline direction) and $22 \mathrm{ft}$ wide. The chamber walls are lined with 18 inch deep fiberglass wedges which provide an anechoic acoustic environment above $175 \mathrm{~Hz}$. Downstream of the test section, the open jet air flow enters a diffuser through a circular collector with acoustic treatment on its flow impingement surface. The diffuser is designed to operate unstalled and, hence, is not a major source.

The jet is supplied with high pressure air from a compressor system capable of delivering $20 \mathrm{lb} / \mathrm{sec}$ of dry air continuously. The air is heated using a propane SUE burner. Flow from the combustor passes through a muffler which attenuates upstream combustion and valve noise. The airflow rate and combustor temperature are regulated by a programmed logic controller (PLC). Airflow is precisely controlled by using a large 4" valve to set the flow rate and a smaller 1" parallel valve for fine control.

\section{B. Rotating Array}

A novel experimental-diagnostic method was used, comprising a non-invasive phased microphone-array technique for measuring hydrodynamic pressure of large-scale turbulent structures in the jet near field. The technique shown, in Figure 1, combines experimental-diagnostic capabilities developed in recent years for subsonic jets through collaborative research involving Caltech, UTRC, and NASA-GRC. The current second generation array hardware devised by UTRC consists of an open frame creating an acoustically "transparent" structure to which a rotating axial array of microphones is attached while a second axial array remains fixed in location (Fig. 1). The axial extent of the microphone array ranges from $\mathrm{X} / \mathrm{D}=0$ to 13 with 11 microphones installed on each array. The first microphone is located at $0.97 \mathrm{Dj}$ from the centerline while the last microphone in the series is located at $2.5 \mathrm{Dj}$. Array microphones are located on a virtual cone separated axially by $1.2 \mathrm{Dj}$ at a spread angle of 7 degrees. A key design intent of this array is to facilitate application to complex nozzle geometries by minimizing the required microphone count, and maximizing adjustability in terms of axial location and streamwise extent. Hence, the downstream measurement domain can be readily extended by lengthening the microphone support rods in the axial direction.

For a given position of the reference array, phase-locked data between the two arrays is acquired with the number of measurement locations for the rotating array being governed by the desired azimuthal modal resolution. With this approach, the modal content at any axial location is determined by Fourier transformation of the two-point azimuthal correlation. The azimuthal resolution is controlled by the mechanical rotation components and can be set to 1 degree increments or smaller if needed. This approach minimizes the number of microphones needed to resolve higher azimuthal modes in multi-chevron nozzles or asymmetric nozzles. Higher order modes can become a source of aliasing error in complex non-circular nozzles, or in jets with shear-layer excitation at higher modes. Therefore, the current array has been designed for fine resolution in the azimuthal direction. Details of the rotating mechanical components are given in References 3 and 7. 


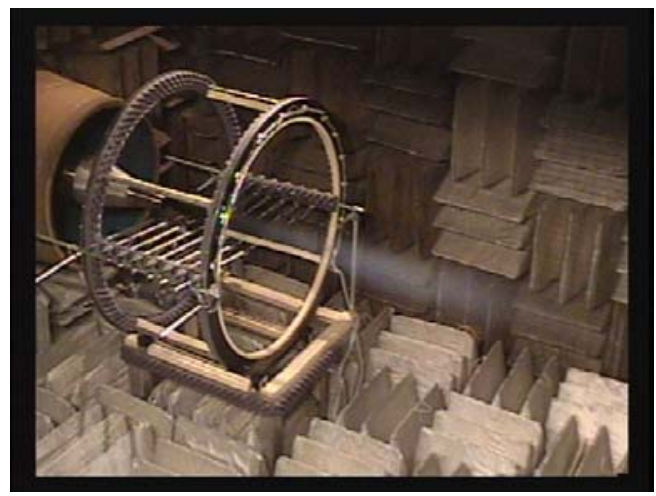

a)

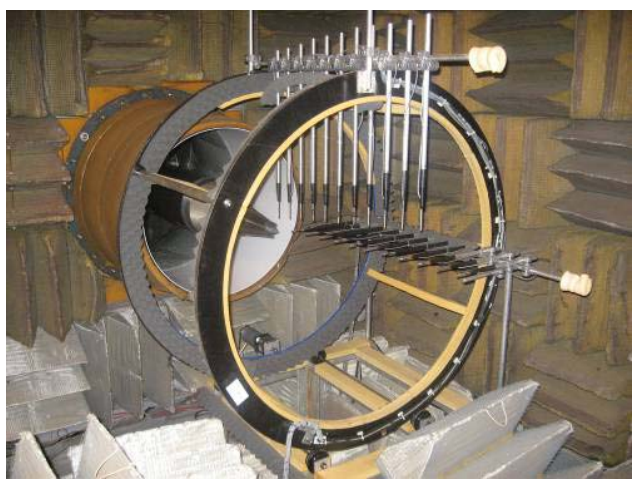

b)

Figure 1. a) Rotating array configuration installed in UTRC Acoustic Research Tunnel

b) view looking upstream into 36 inch diameter open jet wind tunnel with rotating array in vertical plane perpendicular to reference microphones

\section{Nozzles and Flow Fields}

In the current study, a 3" diameter round converging-diverging (CD) supersonic nozzle was designed using a method-of-characteristics to provide ideal expansion or shock free flow at the nozzle exit. Figure 2 shows a schematic of the round nozzle interior contour and a photo of the nozzle mounted at the termination of the ART centerline piping. The screen located upstream of the nozzle in the photo covers the suction system used to control boundary layer thickness on the exterior of the piping leading to the nozzle. A secondary flow pipe used for dual stream jet noise studies is embedded behind the screen although it is capped off in the current configuration.
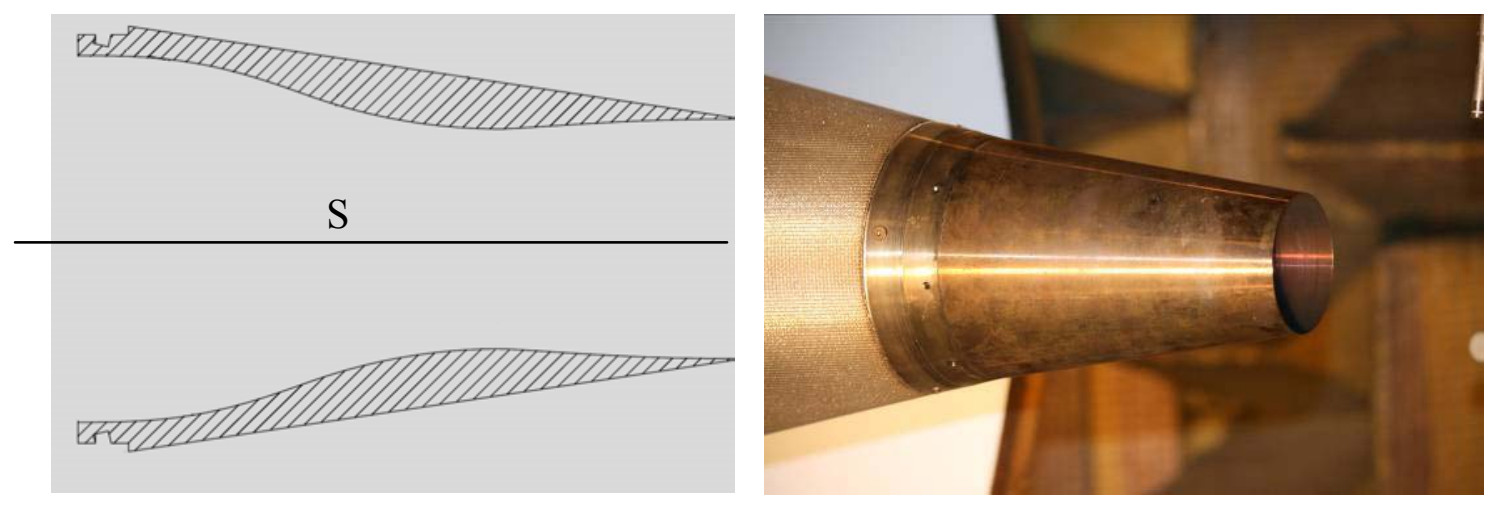

Figure 2. Schematic of $M=1.5 \mathrm{CD}$ nozzle interior and photo installed on ART centerline piping

A CD nozzle geometry was chosen for the baseline supersonic cases to facilitate the detection of organized structures in the jet near field without the presence of shock cells. Off-design measurements were then conducted at nozzle pressure ratios corresponding to over and under expanded conditions. Stagnation temperature ratios (Tr) in the test matrix, relative to a nominal upstream plenum temperature of $72^{\circ} \mathrm{F}$, ranged from cold (unheated) to $\operatorname{Tr}=2.0$. Nozzle exit diameter $\left(D_{j}\right)$ was selected as 3 ".

A six chevron nozzle was also designed as a variant of the $\mathrm{Mj}=1.5 \mathrm{CD}$ nozzle. The chevrons are configured as extensions to the 3" diameter CD nozzle with a one degree angle of penetration into the flow. Figure 3 shows a schematic of the nozzle interior contour and a photo of the chevron nozzle. The nozzle 
equivalent exit area, represented by the projected downstream area, is reduced by $6 \%$ due to the penetration. Figure 4 shows a photo of the chevron nozzle installed on the jet rig centerline with the downstream near field microphones in the field of view.
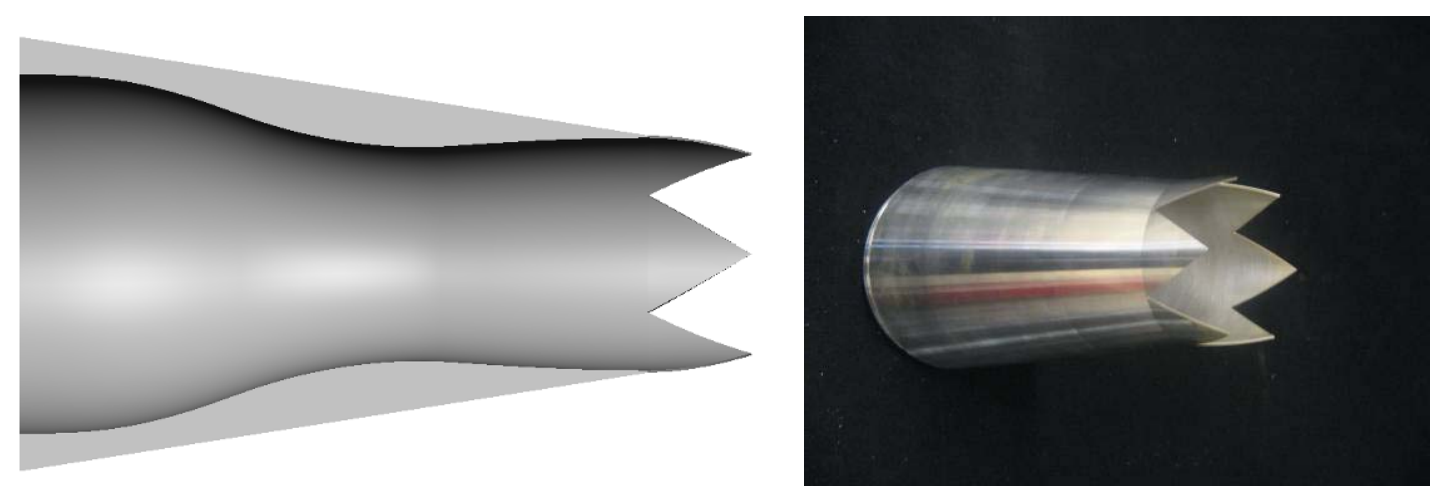

Figure 3. Schematic of $\mathrm{Mj}=1.5$ chevron nozzle interior contour and photo

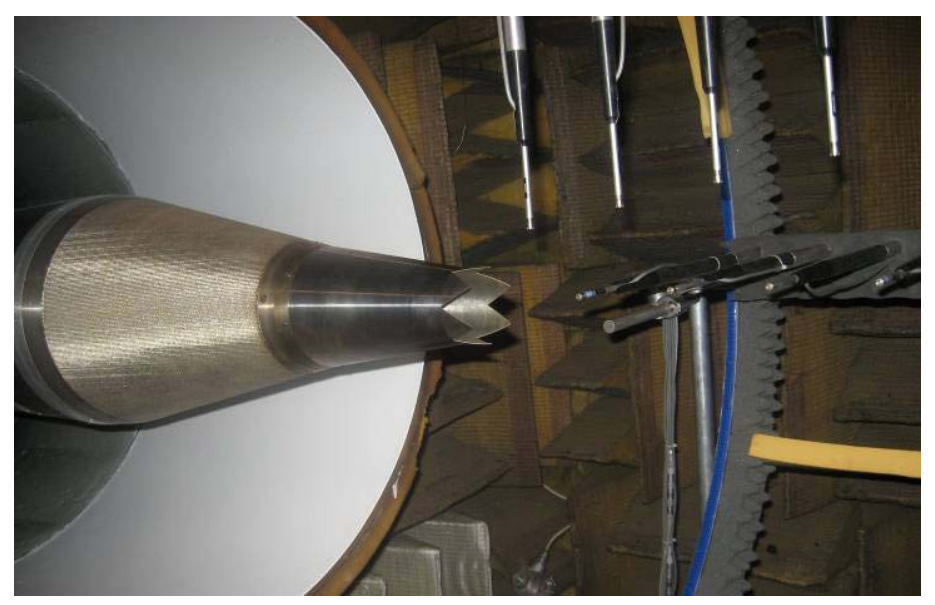

Figure 4. Converging-diverging chevron nozzle with fixed and rotating near field microphones in field of view.

Baseline mean flow data was acquired to document the potential core length and sonic point in the exhaust stream. Radial profiles of total pressure, total temperature, and static pressure were acquired using a multiprobe from which Mach number, static temperature, and velocity were calculated. Surveys were conducted in the horizontal and vertical direction as shown in Figure $\mathbf{5}$ for the round nozzle to confirm that the traverse was aligned with the jet centerline. Centerline decay measurements along the jet axis were also acquired.

Forward flight simulation was not applied in the current study to avoid contaminating the near field microphones with self generated noise due to the ART open jet flow over the microphones. However, due to flow entrainment generated by the nozzle exhaust, the open jet surrounding the jet noise rig was held at a constant $\mathrm{M} \sim 0.1$ forward flight velocity using the downstream facility fan. 

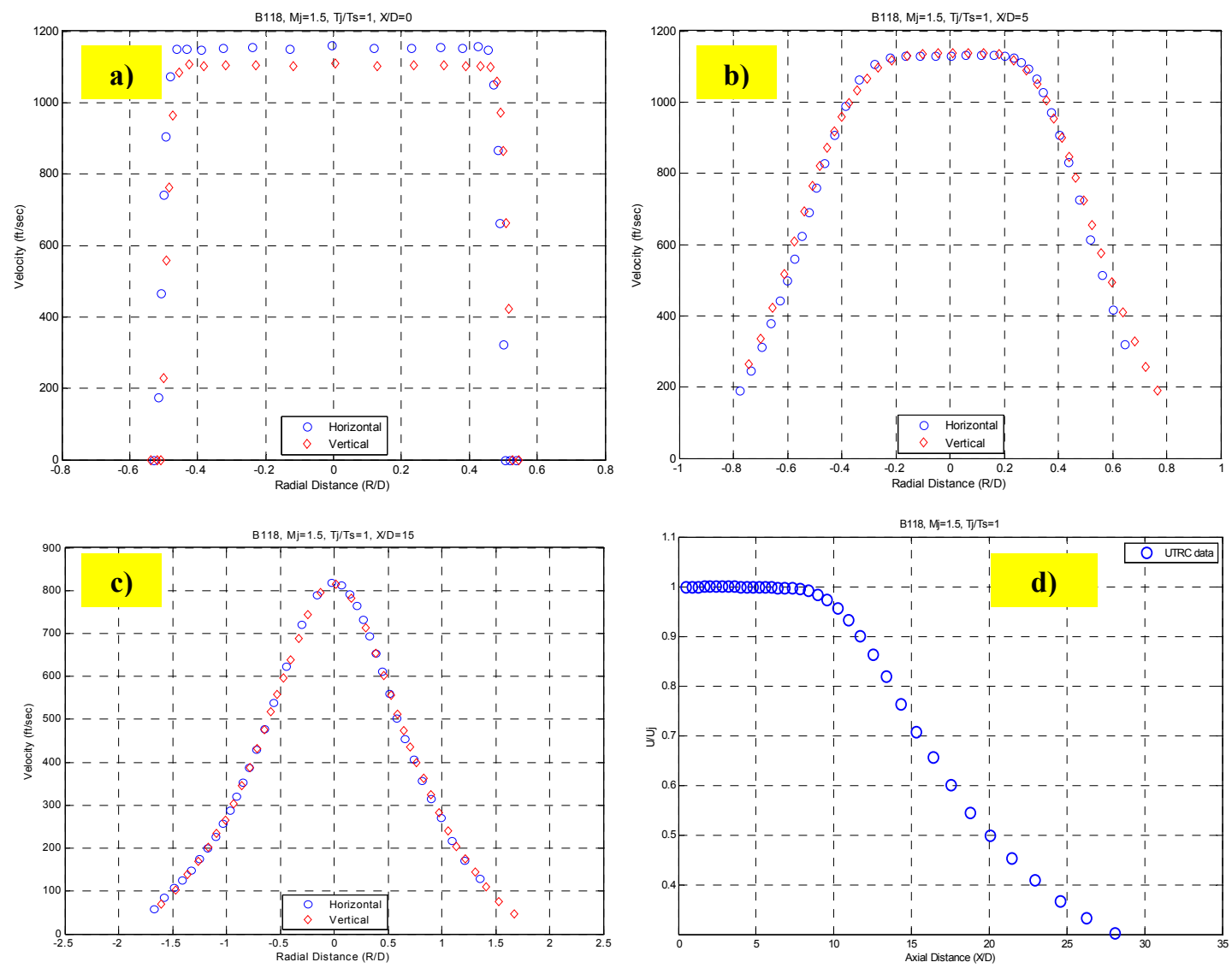

Figure 5: Typical velocity profiles for $M=1.5$ round nozzle, $T r=0.98$ : radial profiles at selected axial stations-- a) $x / D=0, b) x / D=5, c) x / D=15$; axial centerline velocity profile in d)

\section{Instrumentation and Data Systems}

B\&K 1/4" microphones were used for both the near field phased array system and for the far field stations. The microphones were powered using Nexus amplifiers. Simultaneous near and far field data was acquired using a Fanuc DDR-200 digital data recorder. This system is capable of acquiring 64 channels of data simultaneously at rates up to $400 \mathrm{Ksamples} / \mathrm{sec}$ continuously to disk or bursts of data at rates up to 5 Msamples/sec. Most data was acquired at a $100 \mathrm{kHz}$ sampling rate, but selected records were acquired at 200 and $500 \mathrm{kHz}$. The far field microphone data was also recorded at $200 \mathrm{kHz}$ using the standard ART LabView based data acquisition system. All of these sampling rates are well beyond the maximum Strouhal number associated with organized turbulent structures. Real time acoustic data monitoring and analysis was facilitated by dedicated computer clusters connected to the data acquisition systems.

Calibration of the near field and far field microphone systems for amplitude and, in particular phase, was conducted using a B\&K Type 9721 calibrator system developed specifically for UTRC. Amplitude was calibrated to $100 \mathrm{KHz}$ using a B\&K pure tone actuator signal applied to the diaphragm while phase was calibrated to $30 \mathrm{KHz}$ with one microphone used as a reference unit. Near field and far field microphone stations are shown schematically in Figure 6 with measurements located at a sideline minimum distance of 40 jet diameters. 


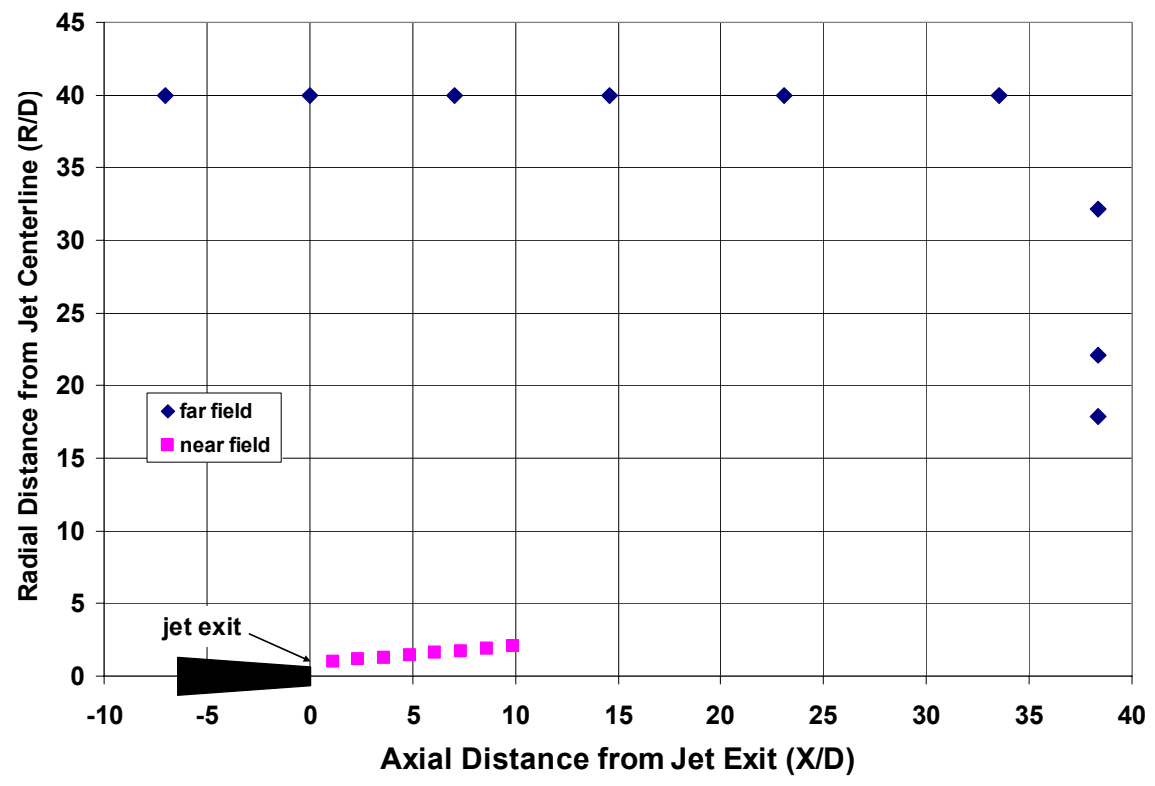

Figure 6. Near field and far field microphone stations

\section{E. Shadowgraph System}

A high-speed, parallel light, direct shadowgraphy system was used to visualize how nozzle geometry affects the shear layer evolution and the large scale structures which are responsible for noise generation. In the case of the chevron nozzle, the shadowgraph system was also used to determine if the chevrons penetrated sufficiently to generate vorticity capable of modifying the flow field. Comparisons between the round and mildly penetrating chevron nozzle provided insight into the jet plume development and a qualitative understanding of the wavepacket modifications observed in the near field hydrodynamic pressure measurements. In the case of off-design operation, the shadowgraph system tracked the chevron suppression of the shock cell structure, thereby, reducing the screech tones.

The shadowgraph system setup shown in Figure 7 consisted of a large retro-reflecting screen located on the opposing side of the supersonic flow allowing much larger flow areas to be visualized compared to using limited size mirrors in a Schlieren system. The rotating array cage shown in Figure 1 was used to support the reflective screen in Figure 7. Near field microphones installed in the rotating cage remained in position providing a direct visualization of the small clearance between the shear layer and the microphones. A high speed camera with 20,000 frames per second and nanosecond exposure times was used to capture the flow field dynamics. The camera was located slightly behind and to one side of the light source. The low light sensitivity of the camera and high spatial resolution allowed the flow to be illuminated with a 500 watt xenon continuous light source instead of relying on expensive, high repetition rate flash units. 

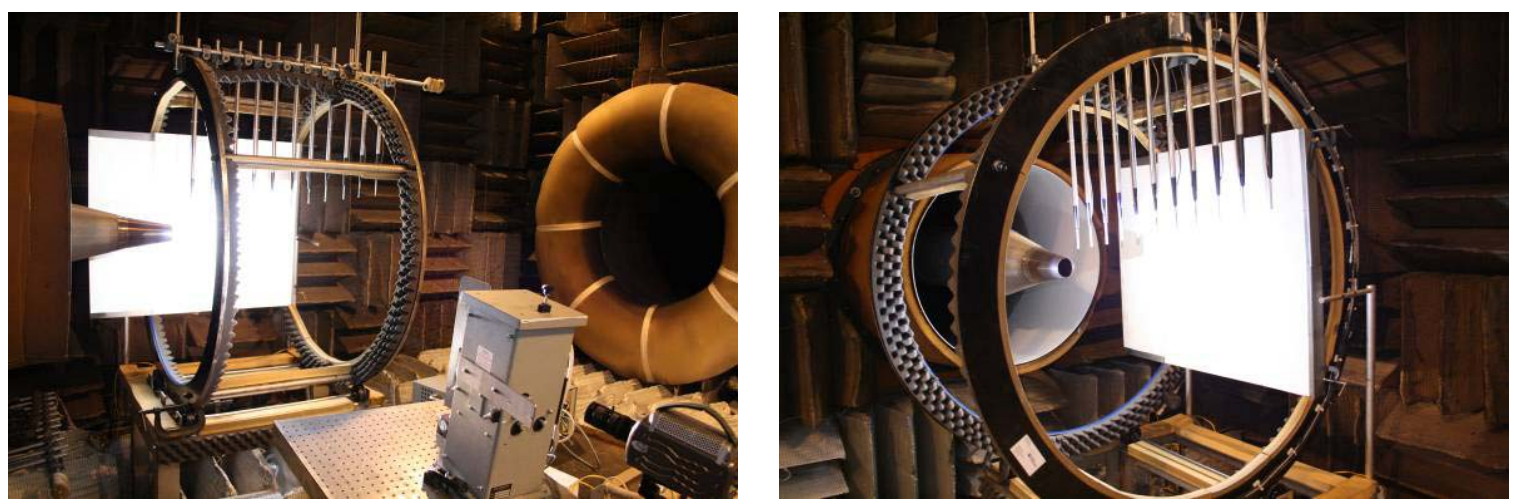

Figure 7. Shadowgraph system installed in ART with large retro-reflective image plane and near field microphone rotating array.

The individual image frames from the camera were post processed to enhance features. This was done using the Matlab $\odot$ image processing toolbox. A background image, obtained with no flow, was subtracted from each video frame. This improves the non-uniform light distribution and highlights the flow. Next, the image is adjusted so that $1 \%$ of image is saturated at high and low intensities, thereby increasing contrast. Finally, an adaptive histogram equalization technique was used to enhance the contrast of the image by transforming the values using contrast-limited adaptive histogram equalization.

\section{F. Small Aperture Linear Array (SALA) System}

To localize source modifications as chevrons and other nozzle geometries are investigated, a small aperture linear array was developed for mapping acoustic source distributions along the jet centerline. A photo of the 22 microphone system is shown in Figure 8a) with the two viewing stations and the included angles shown in Figure 8b).

Emphasis was placed on maintaining a small included angle between the upstream and downstream microphones at each viewing station to minimize the source directivity change across the array. Current beamforming methods and post processing schemes assume an omni-directional source at each axial station. Ideally, this condition can be satisfied if the included angles are sufficiently small such that the source directivity pattern and spectrum features remain constant within the viewing angle. This condition was met at the 130-145 degrees position with an included angle of only 15 degrees and an almost flat directivity pattern at high $\operatorname{Tr}$ values (see Figure 12).

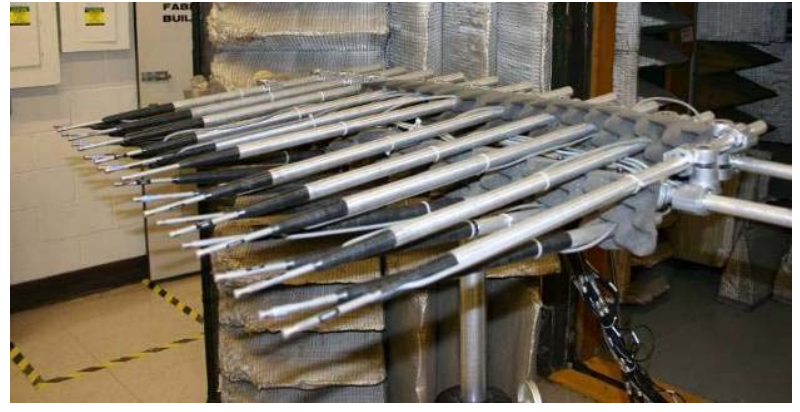

a)

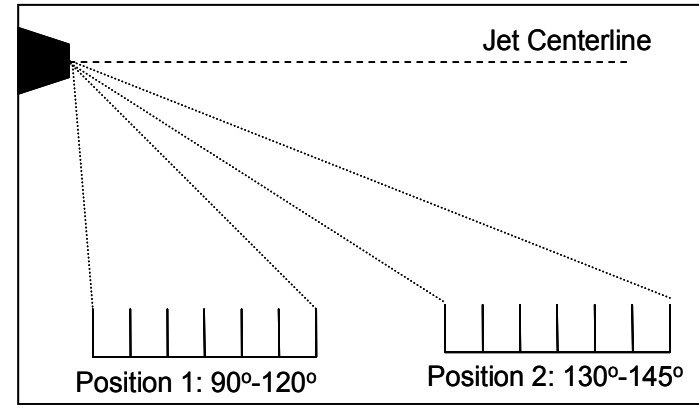

b)

Figure 8. Small Aperture Array System

a) 22 microphone system

b) schematic of array viewing stations and included angles 
The SALA calibration methods and data processing algorithms are described in References 11 and 12. These beamforming methods used an assumed free-space Green's function for noise propagation. Figure 9 shows the point spread function for the array configuration at a sideline distance of 40 diameters from the jet centerline. An axial resolution of approximately one diameter (or less) is possible at high frequencies above $50 \mathrm{KHz}$ to facilitate the detection of the impulsive signature origin. A shear layer correction was not needed given the low $\mathrm{M}=0.1$ condition of the ART open jet flow. Additional post-processing of the beamforming results using DAMAS2 (Ref. 13) was also performed in order to separate the true source field from the array points spread function.

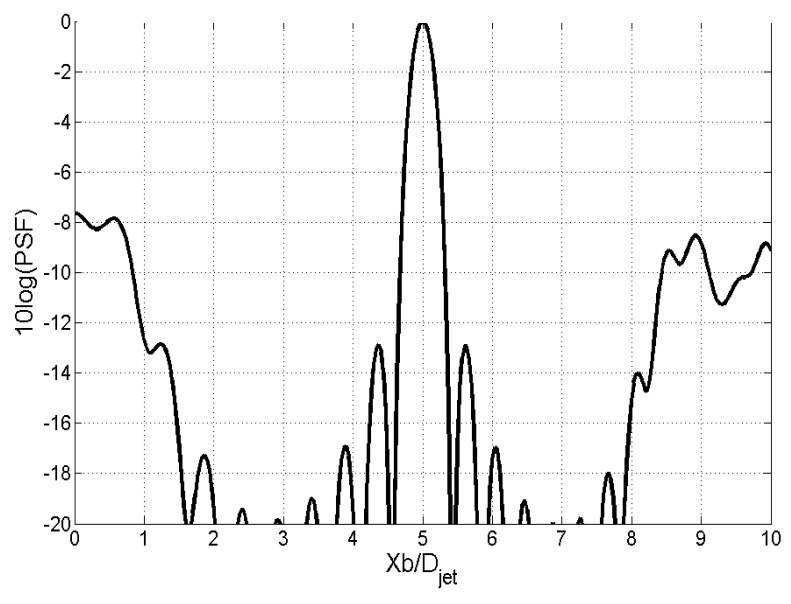

Figure 9. Array Point Spread Function at 50KHz, 40 diameters from jet centerline

\section{Round Nozzle Far Field Acoustic Results}

\section{A. Data Quality and Atmospheric Corrections}

The quality of ART far field data has been shown to be in good agreement (Ref. 7) with acoustic spectra of Tanna (Ref. 14, 15) as well as Brown \& Bridges (Ref. 16) at forward angles where fine scale turbulence dominates and at aft angles where organized structure noise dominates. Atmospheric corrections have been applied to the data accounting for temperature and humidity effects based on the approach developed by Bass, Sutherland, Zuckerman, Blackstock, and D.M. Hester (Ref. 17). Data obtained in the coordinate system shown in Figure 6 is, thereby, converted to Standard Day conditions.

\section{B. Far Field Spectra and Similarity Spectrum Scaling}

The current far field acoustic data base is well suited for developing a semi-empirical noise model for first order jet noise predictions during early design iterations. One possible approach uses the similarity spectra developed by Tam to represent the fine scale turbulence noise (F function) and large-scale turbulence structure noise ( $\mathrm{G}$ function) considered to be the dominant jet noise mechanisms. Recent studies by Tam et al (Ref. 9) have provided experimental evidence and analysis to support this idea and the two resulting similarity functions. The following subsections compare the current round nozzle data base with the spectral, directivity, overall sound pressure level, and velocity dependence characteristics identified by Tam.

Figure 10 shows the $\mathrm{F}$ function fitted to angles between 80 and 130 degrees while the $\mathrm{G}$ function is fitted over the 130 to 155 degrees range for a data set with $\mathrm{Mj}=1.5, \mathrm{Tr}=2.0$ (corresponds to $\mathrm{B} 123$ in Reference 16) The fit, performed at the measured distances in the ART (Figure 6), shows good agreement in both ranges. At 130 degrees, where both functions are shown, the poor fit of the $\mathrm{F}$ function indicates that the $\mathrm{G}$ function dominates. This suggests that the transition between fine scale and large structure noise occurs in the vicinity of 110-120 degrees in alignment with recent directivity assessments by Tam. 
To facilitate the adjustment of similarity spectra over the 80-155 degrees range in Figure 10, an optimization scheme was developed to fit a combined $\mathrm{F}$ and $\mathrm{G}$ function to each of the measured spectra. This provided a smooth transition at angles where spectra shifted from fine scale turbulence noise to large scale structure noise. This technique was applied to spectra spanning the $\operatorname{Tr}=0.75$ to 2.0 range investigated in the current study.
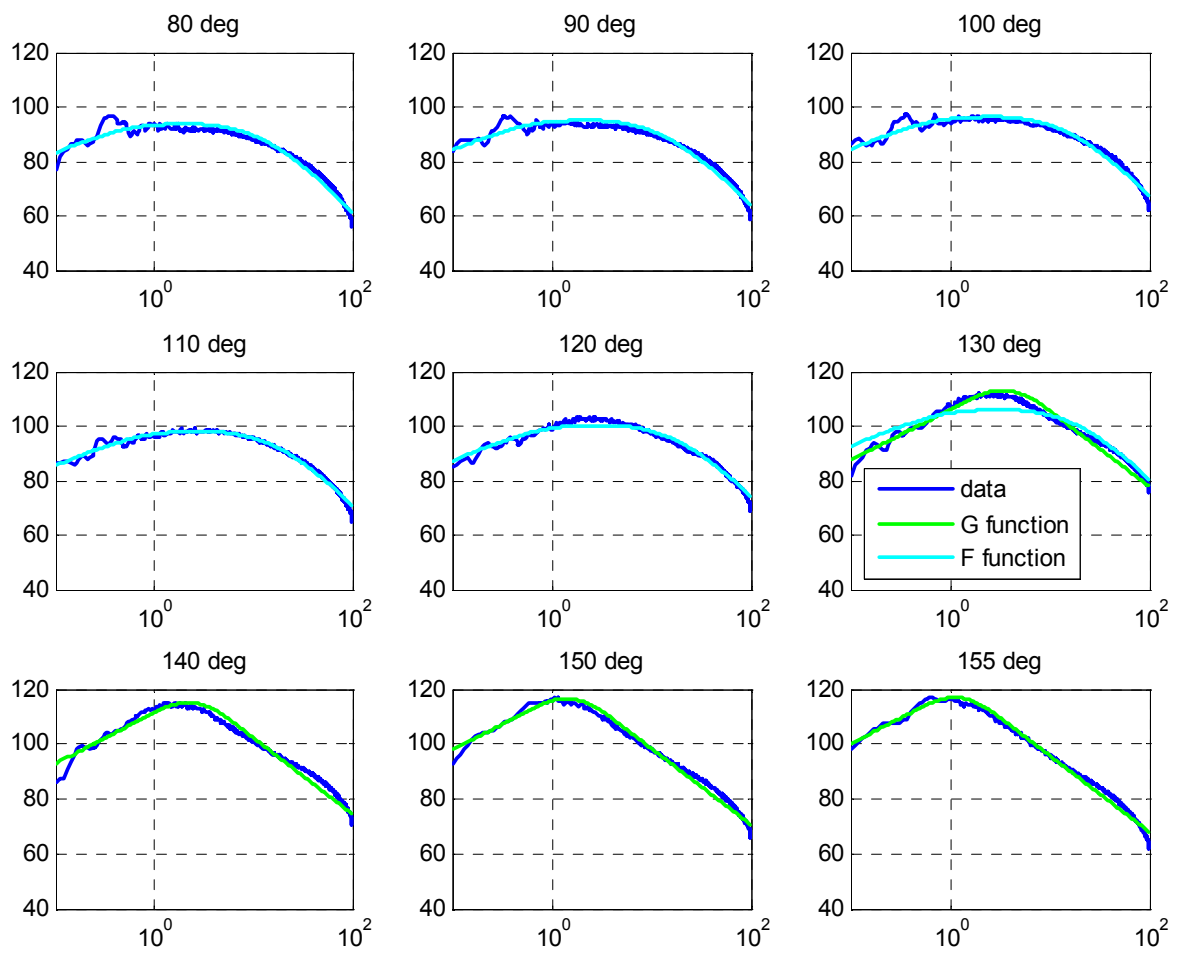

Figure 10. Narrowband spectra vs. directivity with $F$ and $G$ functions fitted to data.

\section{Overall Sound Pressure Level Variation with Directivity and Temperature/Velocity Ratio}

Measured spectra, such as in Figure 10, can be integrated to obtain the OASPL level variation with jet temperature (or jet velocity) for the fixed $\mathrm{Mj}=1.5$ nozzle. Figure 11 shows the resulting OASPL variation with directivity angle for jet temperature ratios ranging from $\mathrm{Tr}=0.75$ to 2.0 (or jet velocity ratio ranging from $\mathrm{Vj} / \mathrm{a}_{\mathbf{0}}=1.3$ to 2.15 ). Two distinct directional characteristics can be extracted from this plot: a) a highly directional source in the downstream quadrant for which the OASPL decreases rapidly in the upstream direction, and b) a non-directional source which increases in OASPL gradually in the downstream direction until it is masked by the directional source. The different directional dependencies, which continue to hold for all $\mathrm{Tr}$ values, support the dual source fine scale and large-scale structure noise mechanism viewpoint of Tam. Also, the occurrence of the transition between the highly directional and non-directional pattern in Figure 11 at $\sim 120$ degrees follows the similarity spectrum transition between fine scale and large-scale structure noise observed at $\sim 120$ degrees in Figure 10 providing additional support for the dual source viewpoint.

Figure 11 can also be converted to a polar coordinate representation as shown in Figure 12 to allow tracking other key features of the fine scale and large-scale structure noise. Based on the latter figure, OASPL levels near 90 degrees increase in equal $\mathrm{dB}$ increments between $\mathrm{Tr}=0.75$ and 2.0 although at a slow rate compared to the aft angles. At aft angles, such as 130 degrees, OASPL levels increase at a higher rate. This characteristic is retained near 150 degrees (with the exception of the highest temperature ratios). The rapid noise increase feature observed here is aligned with the power law reported by Tam (Ref. 9) where 
the exponent " $\mathrm{n}$ " is significantly larger at aft angles than at shallow angles because of the higher efficiency of the organized structure noise. Figure 12 also suggests that the large-scale structure directivity pattern "rotates" in the forward direction as $\operatorname{Tr}$ (or velocity) increases.

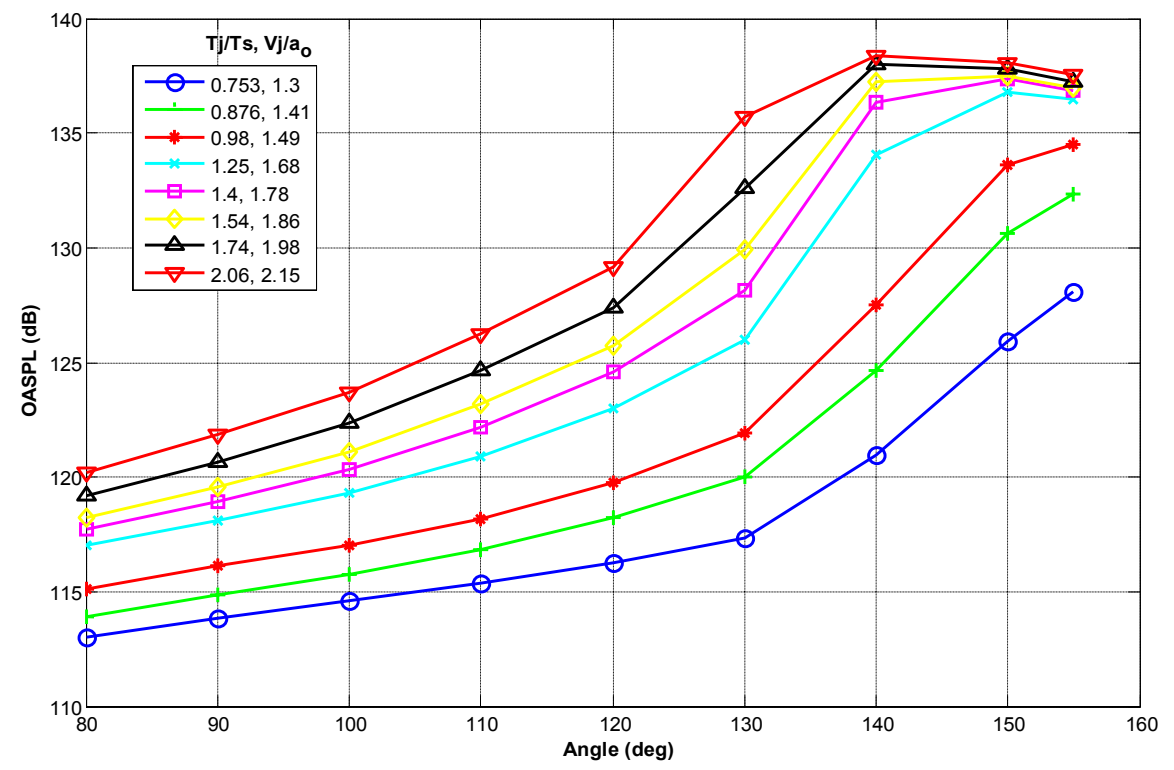

Figure 11. OASPL dependence on directivity angle and temperature ratio, $\mathrm{Mj}=1.5$

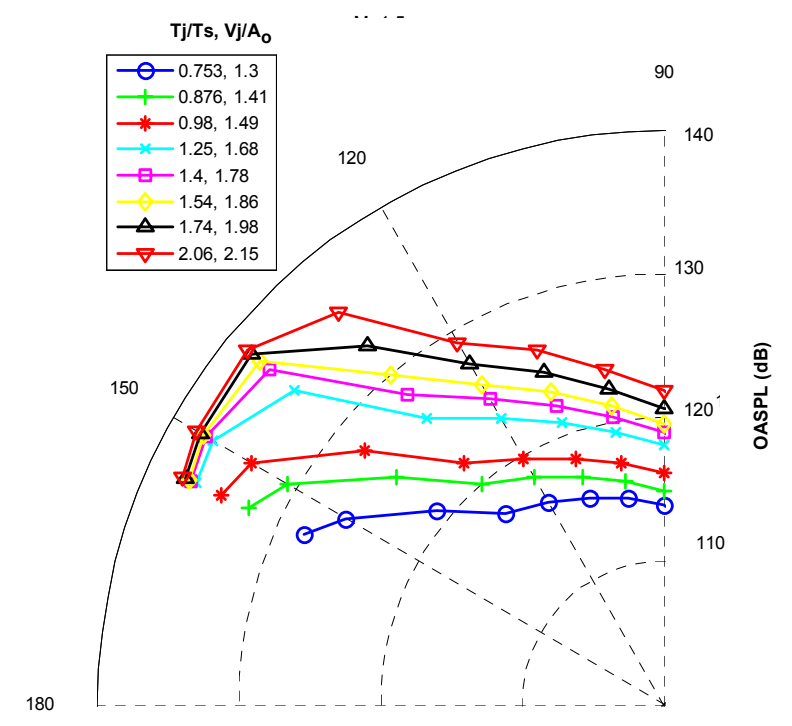

Figure 12. OASPL directivity versus temperature ratio/velocity ratio, $\mathrm{Mj}=1.5$

The large-scale structure noise generation efficiency is not continually retained at the aft angles as already noted for highest $\operatorname{Tr}$ values near 150 degrees in Figure 12. The extreme aft radiation angles display an invariant OASPL with increasing velocity/temperature as indicated by the small $\mathrm{dB}$ increments suggesting a "plateau" in the large scale structure noise generation. This feature is best displayed via Figure 13 with OASPL plotted as a function of jet exit velocity for the fixed $\mathrm{Mj}=1.5$ nozzle. Plotted for comparison is a $\mathrm{V}^{8}$ velocity dependence (red line in the subplots). The onset of the plateau feature, marked by the notation "Break point" in the figure, progressively shifts to lower angles at higher $\operatorname{Tr}$ values in agreement with Figure 12. 
The invariance is accentuated at further aft angles as found by plotting recent data obtained from the study of Brown and Bridges (Ref. 16) conducted at the same Mj and Tr conditions. Their data, replotted in the same format as in Figure 13, is shown here as Figure 14 with angles approaching 162 degrees. The accentuated inefficiency of the organized structure noise is clearly identified here.

A similar invariance was observed for the full scale engine data reported earlier by Schlinker et al. (Ref. 18). At the highest temperature condition for the engine, the velocity scaling law transitioned to $a \sim V^{2}$ dependence at a 135 degrees angle. In this case, the noise increased by $\sim 3 \mathrm{~dB}$ rather than the $9 \mathrm{~dB}$ projected from $\mathrm{V}^{8}$. The dramatically lower noise levels in the high velocity-high temperature condition is possibly due to decreased density at the extremely high exhaust temperatures of the engine. A classical scaling law for density effects estimates a lower noise level based on the ratio of: (engine exhaust density/baseline density) ${ }^{\mathrm{w}}$ where $\mathrm{w}$ is an experimentally determined constant. Since density scales with static temperature, the lower noise level can be estimated using the differences in temperature ratios. Given the temperatures measured in the current engine test, such density effects can explain the transition and plateau effect.
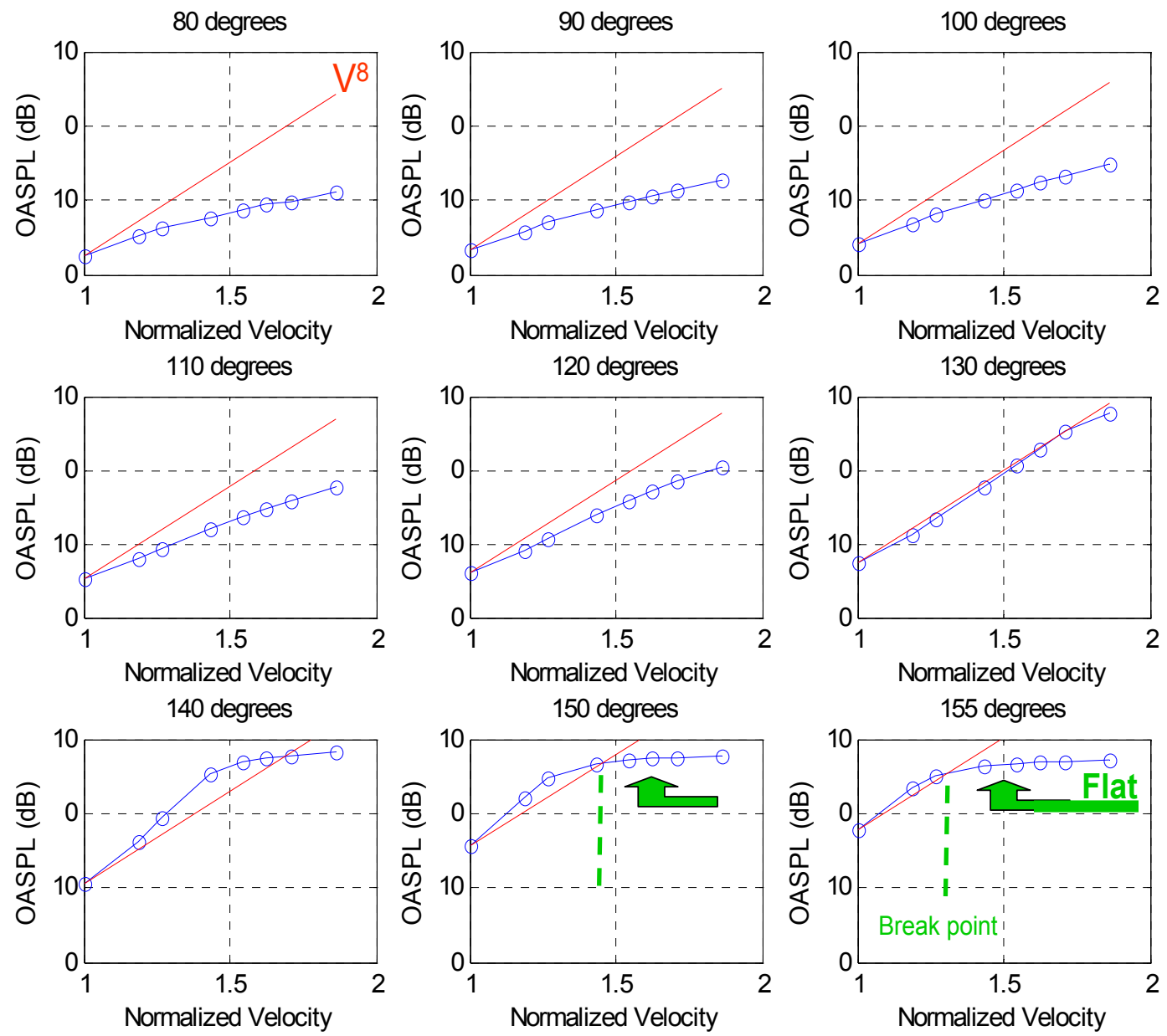

Figure 13. Overall sound pressure level dependence on jet velocity as a function of directivity angle, $\mathrm{Mj}=1.5, \mathrm{Tr}=1.76$. 

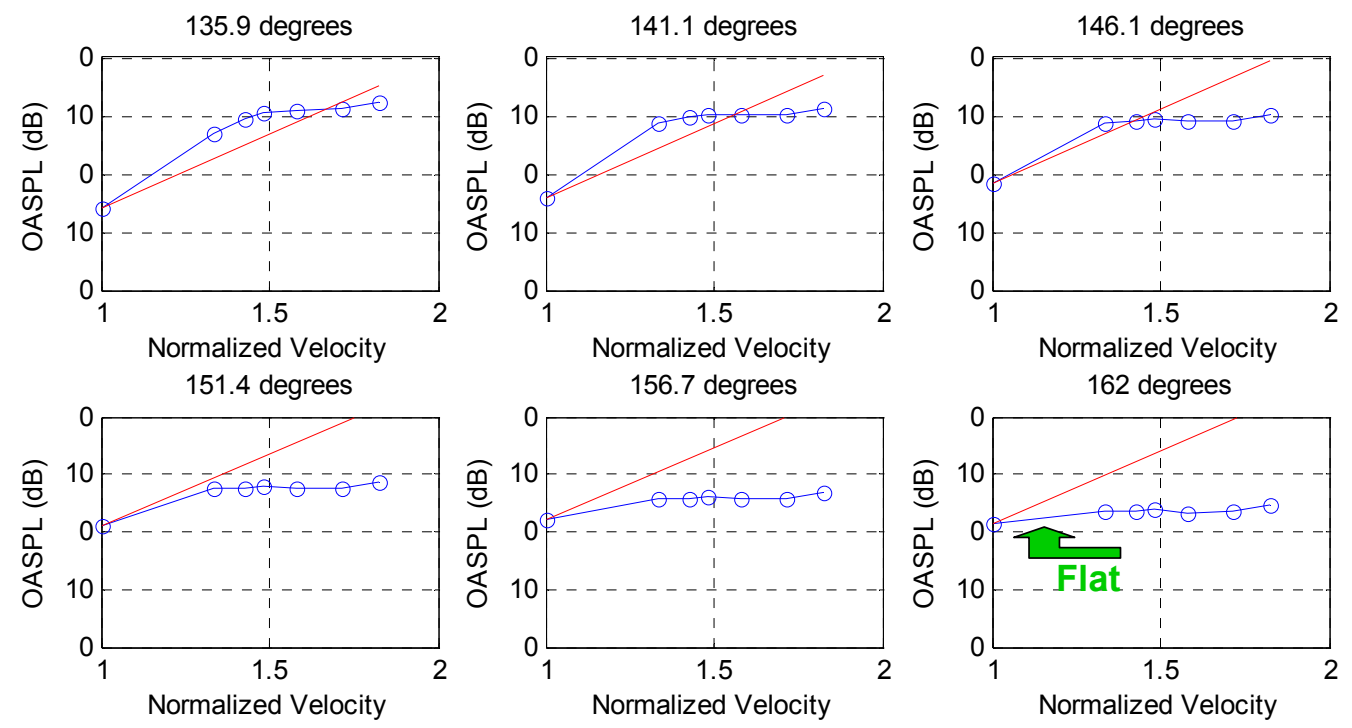

\section{Figure 14. Overall sound pressure level dependence on jet velocity as a function of directivity angle, $M j=1.5, T r=1.76$, raw data from $\operatorname{Ref} 18$.}

\section{Chevron Nozzle Far Field Results}

\section{A. Chevron Noise Reduction at Ideal Expansion}

Figure 15a) compares the baseline $\mathrm{Mj}=1.5$ ideal expansion $\mathrm{CD}$ nozzle narrowband spectra with the mild penetration chevron nozzle at selected directivity angles for operating conditons corresponding to B122 in Reference 14. The spectra indicate $\sim 2 \mathrm{~dB}$ reduction at extreme aft angles for frequencies beyond the spectral peak. High frequency noise increases typical of chevron designs, are absent. At the 130 degrees directivity angle the chevron impact was negligible while at forward angles, broadband noise increased by $\sim 3-4 \mathrm{~dB}$. Figure 15b) presents the delta between the round and chevron nozzle spectra in terms of $1 / 3$ octave band noise reductions. It should be noted that the spectra were insensitive to whether a chevron peak or trough was oriented towards the far field microphone array.

The small chevron reduction at aft angles without an associated noise penalty was considered sufficient to test the chevron impact on the jet near field array measurements of the organized structure wavepackets. A future paper will focus on the modal decomposition of the chevron mitigated wavepackets allowing the current paper to highlight the chevron impact on the statistical characteristics of the acoustic far field.

\section{B. Chevron Suppression of Screech Tones}

The primary benefit of the chevron geometry was observed at underexpanded off-design conditions as shown in Figure 16a) by the round versus chevron nozzle spectrum comparison at 80 degrees. The underexpanded case represents a jet operating at nozzle pressure ratio corresponding to $\mathrm{Mj}=1.4$. The $1 / 3$ octave band noise reduction in Figure 16b), representing the delta between the round and chevron nozzle, indicates a significant screech tone reduction for this mild penetration chevron design. Thus, for situations requiring aggressive screech tone mitigation, a minimally invasive chevron design can be effective. Although the nozzle discharge coefficient was not measured or predicted here, the mild penetration presumably also has minimal impact on nozzle performance. 

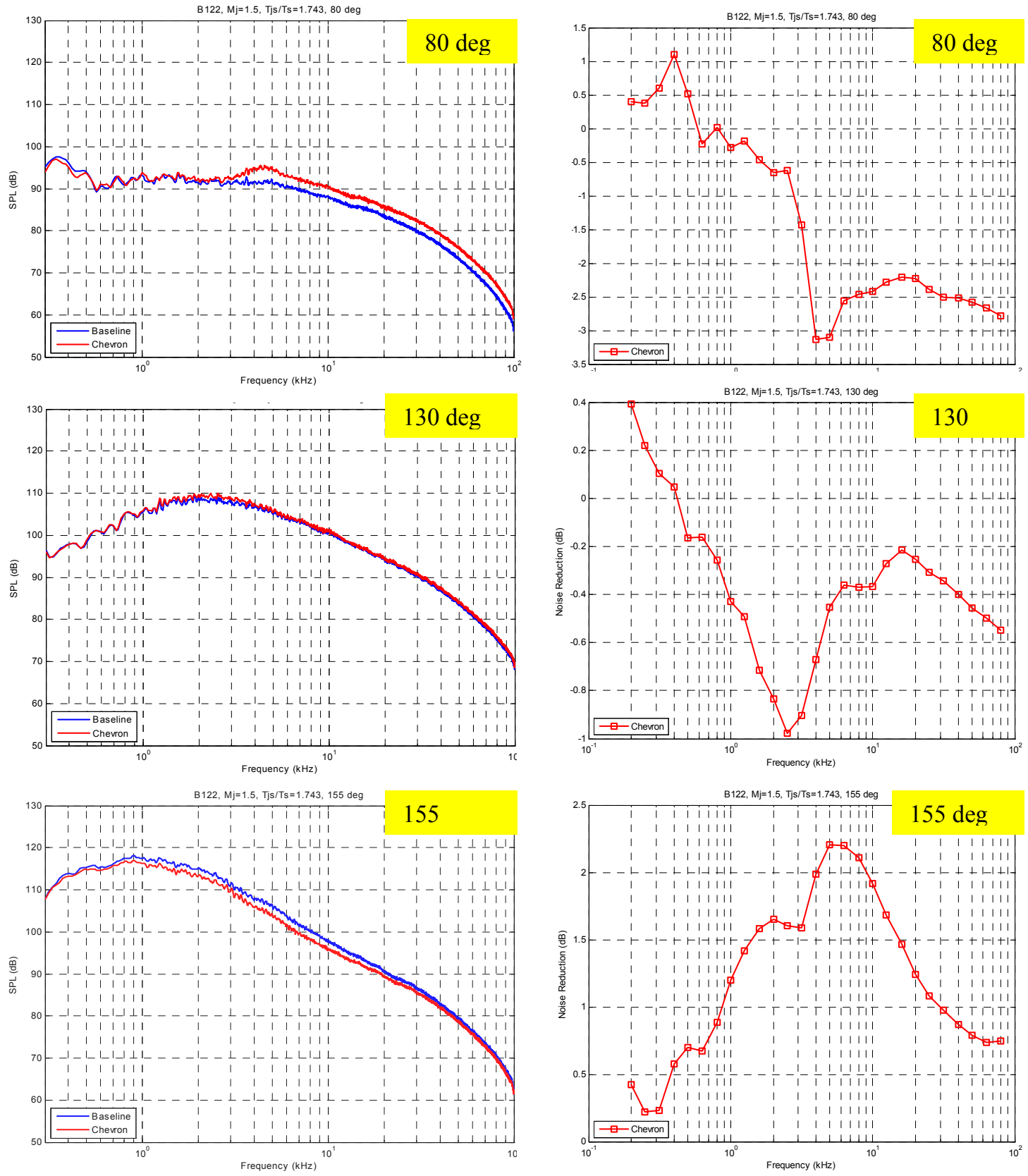

a)

b)

Figure 15. Baseline round vs. chevron nozzle as a function of directivity angle, $\operatorname{Tr}=1.74$

a) narrowband spectra

b) $1 / 3$ octave band noise reduction (reduction plotted as positive value) 


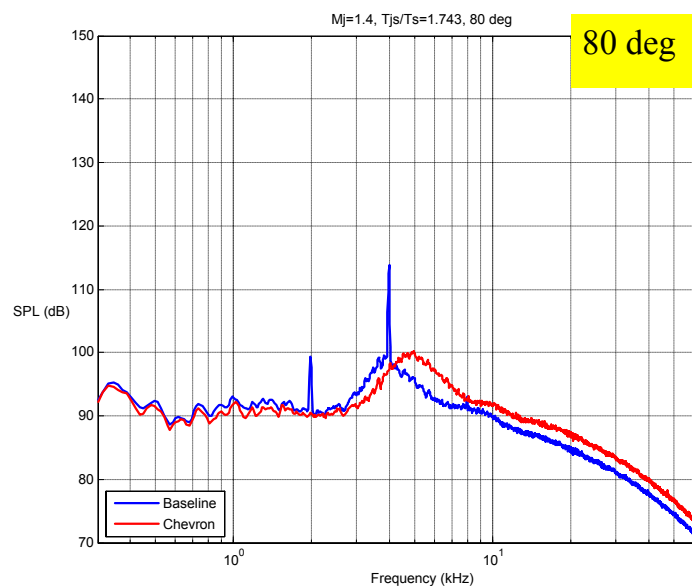

a)

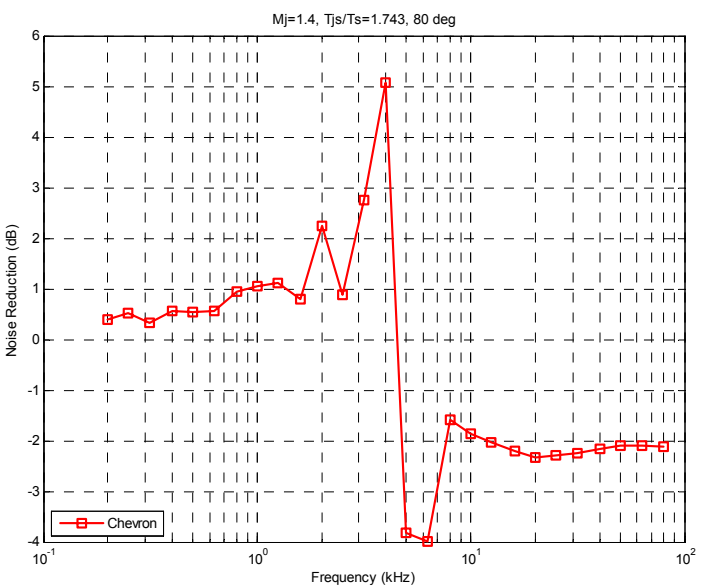

b)

Figure 16. Baseline round vs. chevron nozzle at $80 \mathrm{deg}, M \mathrm{j}=1.4$ (underexpanded), $\operatorname{Tr}=1.74$

a) narrowband spectra

b) $1 / 3$ octave band noise reduction (reduction plotted as positive value)

\section{Chevron Impact on Flow Field}

The shadowgraph system was used to provide a first level evaluation of how nozzle geometry affects the shear layer evolution and the large scale structures which are responsible for noise generation at aft angles. In the case of the chevron nozzle, the shadowgraph system was used to determine if the chevrons penetrated sufficiently to generate vorticity capable of modifying the flow field. Comparisons between the round and mildly penetrating chevron nozzle provided insight into the jet plume development and a better understanding of the wavepacket modifications observed in the near field hydrodynamic pressure measurements. In the case of off-design operation, the shadowgraph system tracked the chevron suppression of the shock cell structure.

Figure 17 shows several shadowgraph images of the round and chevron nozzle. Included in the image are the outlines of the near field microphones. Due to the non-coincident light and camera positions, each microphone shows up twice. The right side image of each pair is the microphone while the left image represent the microphone shadow on the retro-reflective image surface.

Figure 17b) and 17c) present the chevron nozzle in two different clocked orientations. The latter figure shows the chevron generated vorticity "streaks" extending downstream from both the upper and lower chevrons surfaces. A distinctive difference in the shear layer spreading rate is also evident between the round nozzle in a) and the chevron nozzle in b). The close proximity of the near field microphones to the shear layer is also evident demonstrating how the current acoustic array is capable of sensing the convected hydrodynamic pressure field wavepackets which represent the organized structures responsible for large

scale turbulence noise generation. It should be noted that the pressure field wavepackets are not necessarily synonymous with the large structures observed in the shear layer of Figure 17 since the latter features are characteristic of the flow field density gradients as highlighted by the shadowgraph method. Thus, shadowgraph images of large scale structures at the shear layer interface are not necessarily correlated with the convected pressure wavepackets representing organized structure noise. 


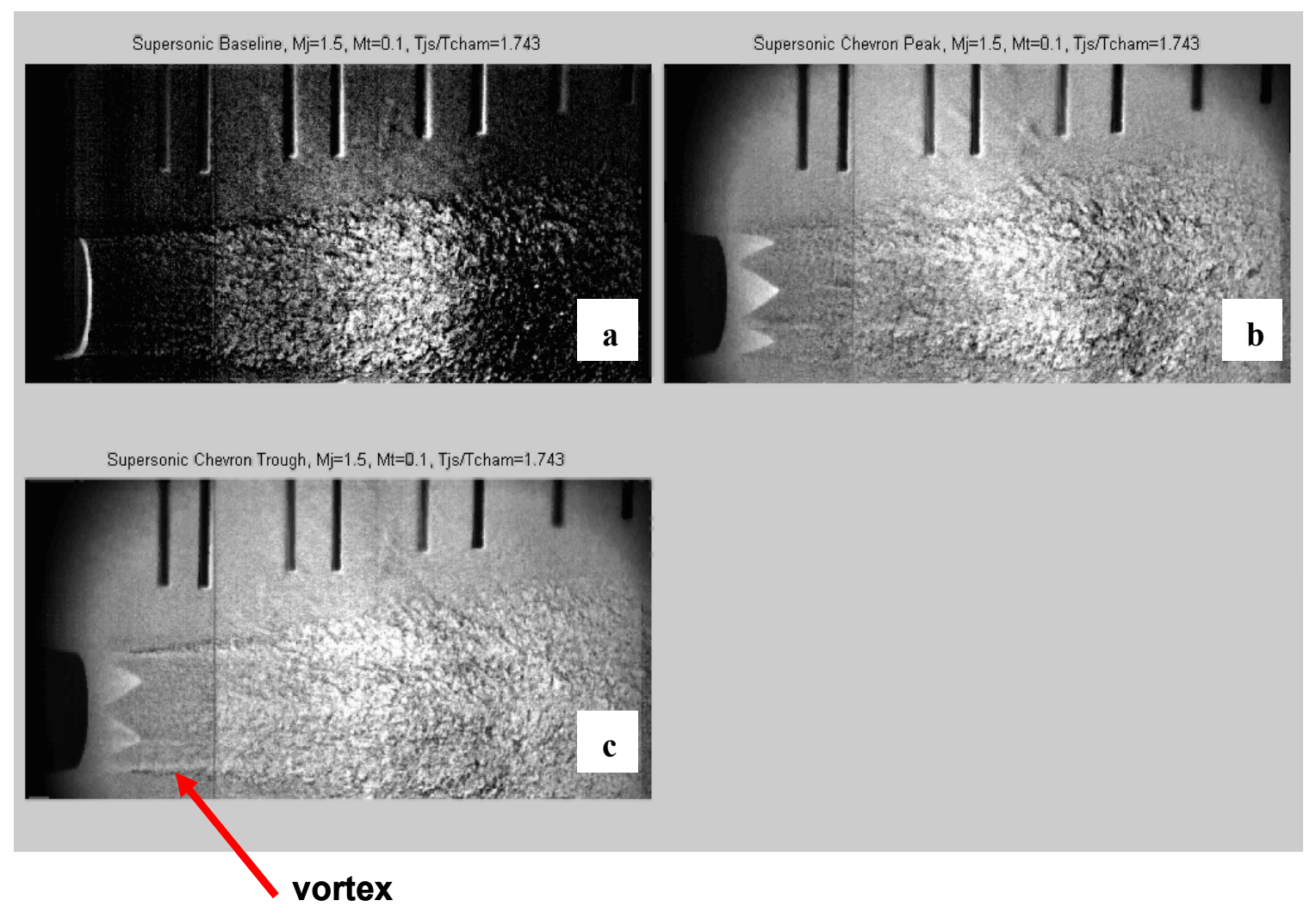

Figure 17. Shadowgraph view of nozzle flow field at $M=1.5, \operatorname{Tr}=1.74$

a) baseline round nozzle

b) chevron nozzle with trough facing camera

c) chevron nozzle with peak facing camera

\section{Acoustic Source Distribution}

\section{A. Small Aperture Array Source Maps}

The axial source distribution responsible for noise radiating at peak directivity angles is presented in Figure 18 based on the post processed results from the Position 2 (130-145 degrees) small aperture array measurement station cited in Figure 8b). This station also coincides with the dominant radiation direction expected from the large-scale turbulent structure noise mechanism. Results are shown for the $\mathrm{Mj}=1.5$ ideal expansion condition plotted in terms of Strouhal number and axial station normalized by jet diameter, i.e. $\mathrm{x} / \mathrm{D}$. At the lowest $\mathrm{Tr}=0.98$ corresponding to a velocity ratio of $\mathrm{Uj} / \mathrm{a}_{0}=1.4$ the jet noise source region is spread over a range of $x / D \sim 7$ to 15 . As velocity increases to $U j / a_{0}=1.78(T r=1.4)$ and finally $U j / a_{0}=2.15$ $(\mathrm{Tr}=1.74)$ the axial source distribution becomes slightly more compact and shifts upstream. During this progression the high frequency decay rate at stations close to the nozzle exit (x/D 4-5) changes from a shallow slope to steeper slope at the highest temperature ratio.

Based on nozzle centerline velocity survey in Figure 5d) the potential core for the $\operatorname{Tr}=0.98$ condition extends to $\mathrm{x} / \mathrm{D} \sim 8-9$ while the sonic point occurs at $\mathrm{x} / \mathrm{D} \sim 15$. Consequently, the dominant source region, corresponding to $\mathrm{x} / \mathrm{D} \sim 7-15$ at this condition, resides downstream of the potential core but upstream of the subsonic regime. It was already noted above that as velocity increases the axial source distribution becomes slightly more compact and shifts upstream in alignment with the expected trend of a shorter potential core. The $\mathrm{x} / \mathrm{D} \sim 7-15$ region also coincides with near field phased array measurements of the large scale turbulence structure acoustic source strength distribution reported in the companion paper (Ref. 4) as will be described below. 


\section{B. Hydrodynamic Pressure Field Source Strength}

Figure 19 provides a schematic of the pressure cross spectrum, $\mathrm{R}_{\mathrm{m}}$, at a given azimuthal mode number. This result was extracted from the convected hydrodynamic wavepacket detected by the near field array measurement technique shown in Figure 1 and analyzed using the methods reported in References 3 and 4. The orthogonal coordinate system shown here represents the $R_{m}$ values mapped out by the rotating array as it covers the axial and azimuthal directions. Calculating the auto spectral density of this signature gives the mean square pressure of the wave packet amplitude along the jet axis. Figure $\mathbf{2 0}$ shows the mean square pressure at each microphone station normalized by the peak amplitude and plotted as a function of $\mathrm{x} / \mathrm{D}$. Here the normalized near field wavepacket pressure amplitudes are presented for azimuthal mode numbers $\mathrm{m}=0,1$ and two selected Strouhal numbers: $\mathrm{St}=0.2$ and 0.4. Results are shown for a subsonic converging nozzle with $\mathrm{Mj}=0.98$ and $\mathrm{Tr}=1.76$ in addition to the ideal expansion supersonic test condition $\mathrm{Mj}=1.5, \mathrm{Tr}=1.74$. Projection of these pressure signals to the acoustic far field (Ref. 4) shows good agreement with directly measured noise levels at aft directivity angles. This demonstrates that the large scale turbulence structure wavepackets control the aft directivity.

In the supersonic case in Figure 20, the wave packet amplitude distribution indicates the dominant source region spans the range $\mathrm{x} / \mathrm{D} \sim 5-14$ based on amplitudes larger than $50 \%$ of the peak value. This region coincides with the far field phased array measurement, described earlier, which identified the dominant source image region as $\mathrm{x} / \mathrm{D} \sim 7-15$. This agreement further supports the cause-effect link between largescale structures in the shear layer and their dominant contribution to aft radiated far field noise; a connection established in Reference 4 and further supported by the results reported here.
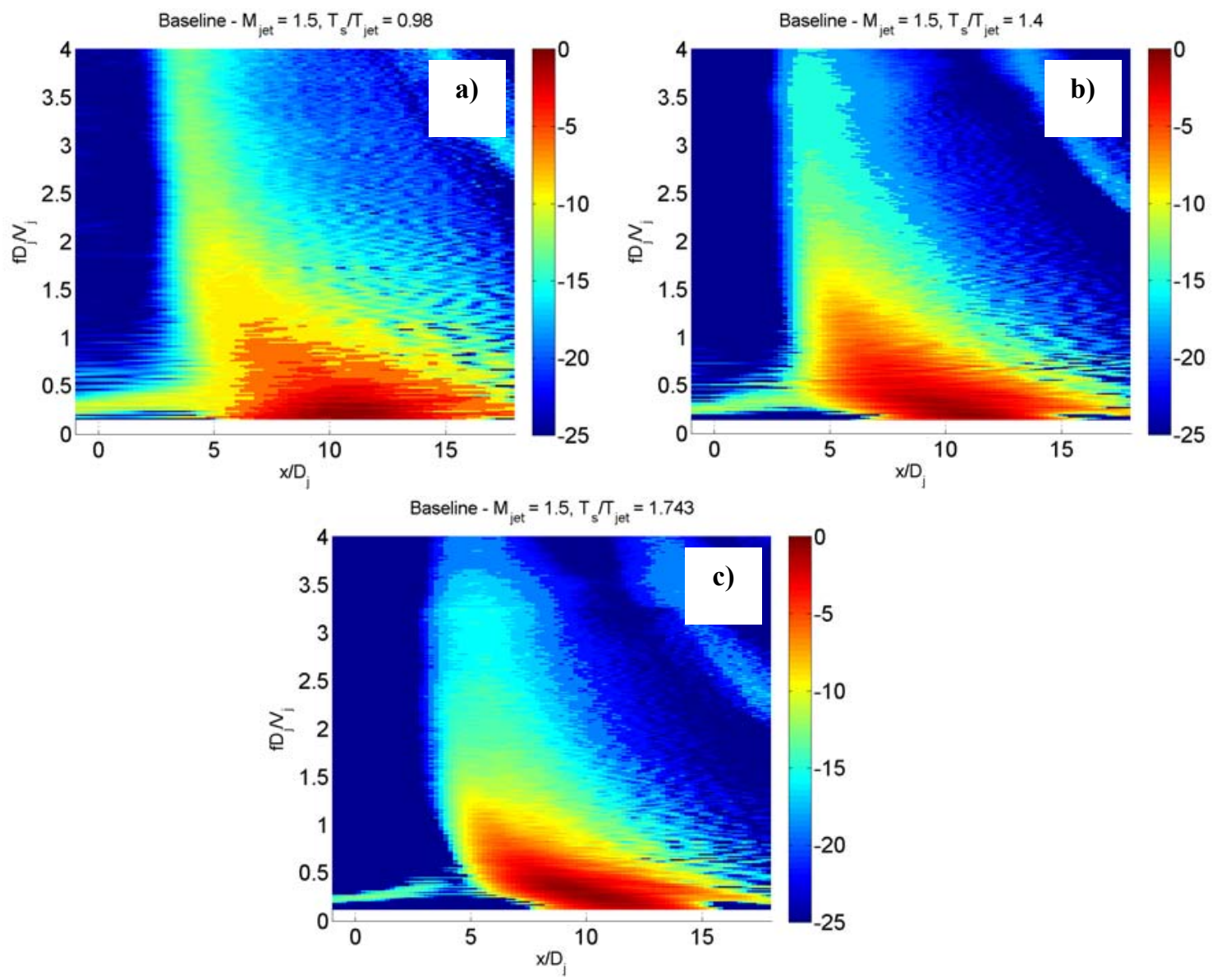

Figure 18. Axial source distribution vs. Strouhal number for ideal expansion $M=1.5$ round nozzle with temperature ratio conditions: a) $\operatorname{Tr}=0.98$, b) $\operatorname{Tr}=1.4$, c) $\operatorname{Tr}=1.74$ 


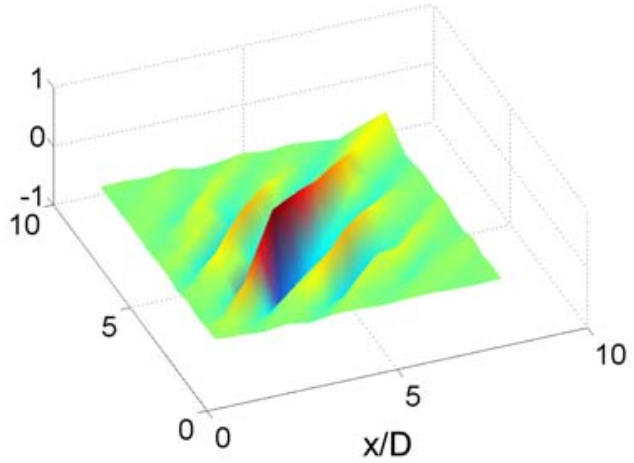

Figure 19. Schematic showing real part, $R_{m}$, of near field array cross spectrum

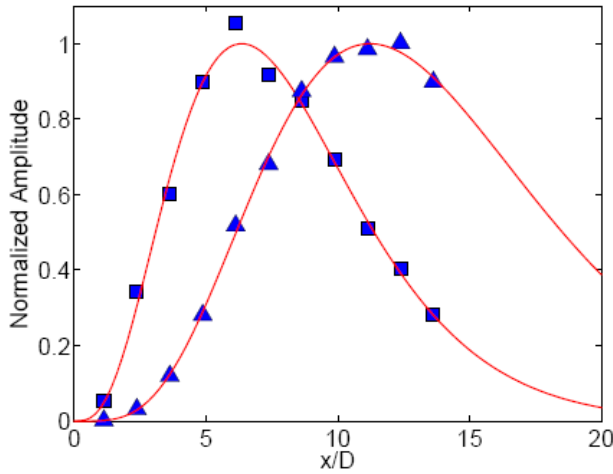

(a) $\mathrm{m}=0 ; \mathrm{St}=0.20$

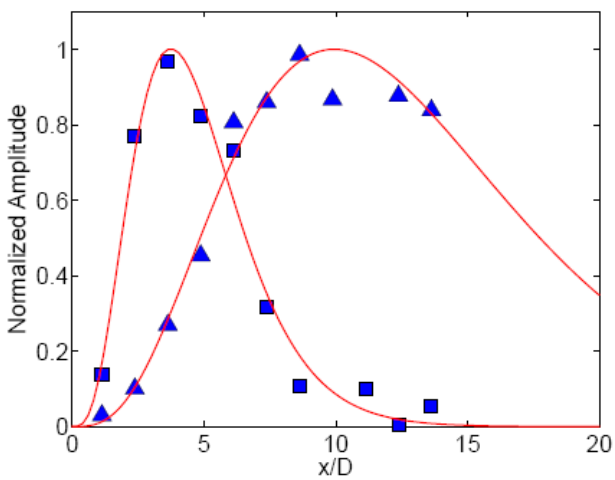

(c) $\mathrm{m}=1 ; \mathrm{St}=0.20$

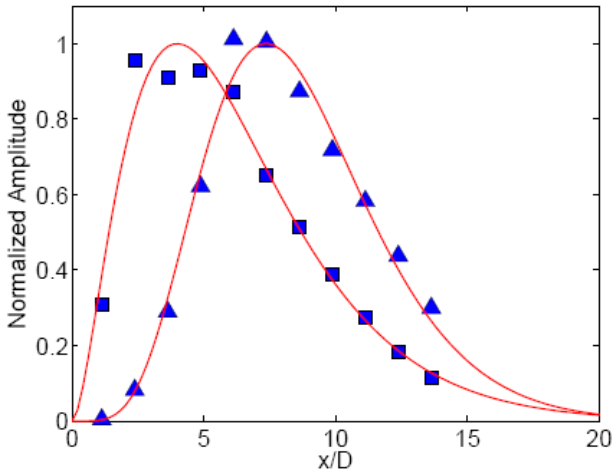

(b) $\mathrm{m}=0 ; \mathrm{St}=0.40$

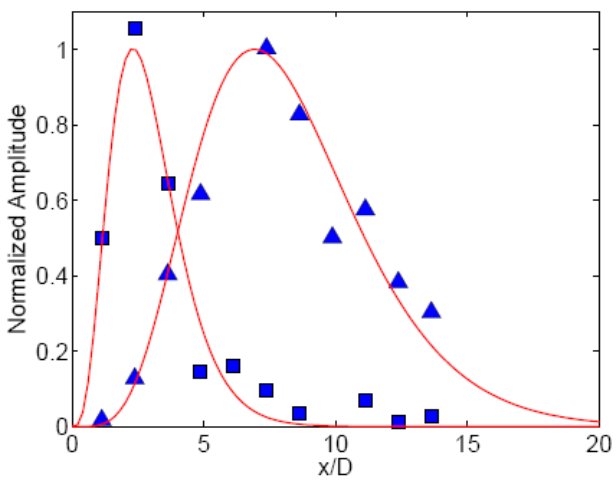

(d) $\mathrm{m}=1 ; \mathrm{St}=0.40$

Figure 20. Measured wave packet amplitude along array (symbols) compared to analytical model (solid lines) for a subsonic jet (triangles) and the supersonic $\mathrm{M}=1.5$, Tr=1.74 CD nozzle (squares). 


\section{Summary}

A comprehensive program is in progress to understand and reduce supersonic exhaust jet noise by controlling instability wave generated large-scale turbulence structures in the jet shear layer, generally accepted to be the source of the dominant aft-angle jet noise. The team is focused on conducting research studies using experimental diagnostics and unique instability modeling suited for identifying control strategies to reduce the large scale structure noise. The current study benchmarks the jet noise from supersonic nozzles designed to provide the supporting experimental data and validations of the modeling elements.

Laboratory scale jet noise experiments are presented for a Mach number of $\mathrm{Mj}=1.5$ with stagnation temperature ratios ranging from $\operatorname{Tr}=0.75$ to 2 . Both round and chevron nozzle data are reported at ideal expansion and off design conditions. Details of diagnostic shadowgraph systems and source localization arrays are described.

The resulting data base provides an opportunity to benchmark the statistical characteristics of round and chevron nozzle data. Far field measured spectra were found to agree with Tam's fine scale and large-scale structure noise similarity spectra. OASPL directivity patterns identified a highly directional source in the downstream quadrant and a non-directional source at 90 degrees to the inlet axis with the transition occurring at $\sim 120$ degrees. This feature, again, is aligned with the dual jet noise source viewpoint. OASPL levels were found to increase slowly with velocity at 90 degrees compared to a rapid increase at downstream angles. $\mathrm{A} \mathrm{V}^{8}$ dependence was observed at 130 degrees while a lower exponent exists at upstream angles indicating that large-scale structures are more efficient sources compared to fine scale turbulence noise. A plateau was observed in the OASPL vs. velocity dependence at extreme aft angles; a feature observed in data sets obtained from other researchers and full scale engine tests. The invariant velocity dependence may be due to density effects although no specific theory or modeling exists to confirm this effect.

Mild penetration chevrons were found to reduce noise levels by $\sim 2 \mathrm{~dB}$ in the aft quadrant at frequencies above the Strouhal peak. However, broadband noise increased at 90 degrees and forward angles. The primary benefit of the chevron geometry was at underexpanded operating conditons with a significant reduction of screech tone amplitude. Thus, for situations requiring aggressive screech tone mitigation, a minimally invasive chevron design may be effective. A high speed shadowgraph system was successful at visualizing how nozzle geometry affects the shear layer evolution and the large scale structures. In the case of the chevron nozzle, the shadowgraph system was used to determine if the chevrons penetrated sufficiently to generate vorticity capable of modifying the flow field.

Based on source images obtained from a far field narrow aperture array, the dominant source region responsible for noise radiating to aft directivity angles resides between the end of the potential core and the sonic point. This region was found to agree with the peak amplitude location of the jet near field pressure wave packets measured using a unique near field array. This observation substantiates the cause-effect link between large-scale structures in the jet shear layer and their dominant contribution to aft radiated far field noise.

\section{Acknowledgements}

The authors gratefully acknowledge NAVAIR and TTC Technologies Inc. for supporting this effort. 


\section{References}

[1] Schlinker, R.H., Reba, R.A., Simonich, J.C., Colonius, T., Gudmundsson, K., and Ladeinde, F., "Toward Prediction and Control of Large Scale Turbulent Structure Supersonic Jet Noise," Proceedings of the ASME Turbo Expo 2009, Orlando, Florida, June 2009, GT2009-60300.

[2] Gudmundsson, K., Colonius, "Nonlinear Parabolized Stability Equation Models for Turbulent Jets and their Radiated Sound," $15^{\text {th }}$ AIAA/CEAS Aeroacoustics Conference, Miami, Florida, May 2009, AIAA 2009-3380.

[3] Reba. R, Simonich, J., and Schlinker, R.H., "Measurement of Source Wave-Packets in High-Speed Jets and Connection to Far Field," $14^{\text {th }}$ AIAA/CEAS Aeroacoustics Conference, Vancouver, Canada, May 2008, AIAA 2008-2891.

[4] Reba, R., Simonich, J.C. and Schlinker, R.H., "Sound Radiated by Large-Scale Wave Packets in Subsonic and Supersonic Jets," $15^{\text {th }}$ AIAA/CEAS Aeroacoustics Conference, Miami, Florida, May 2009, AIAA 2009-3256.

[5] Reba, R.A., Narayanan, S., Colonius, T. and Suzuki, T., "Modeling Jet Noise from Organized Structures Using Near Field Hydrodynamic Pressure", $11^{\text {th }}$ AIAA/CEAS Aeroacoustics Conference, Monterey, CA, May 2005, AIAA 2005-3093.

[6] Suzuki, T. and Colonius, T., "Instability Waves in a Subsonic Round Jet Detected using a Near-Field Phased Microphone Array,” J. Fluid Mech., Vol. 565, pp. 197-226, 2006.

[7] Schlinker, R.H., Simonich, J.C., Reba, "Decomposition of High Speed Jet Noise: Source Characteristics, and Propagation Effects," $14^{\text {th }}$ AIAA/CEAS Aeroacoustics Conference, Vancouver, Canada, May 2008, AIAA 2008-2890.

[8] Tam, C. K. W., Golebiowski, M. and Seiner, J. M., "On the Two Components of Turbulent Mixing Noise from Supersonic Jets," $2^{\text {nd }}$ AIAA/CAES Aeroacoustics Conference, State College, PA, May 1996, AIAA 96-1716.

[9] Tam, C.K.W., Viswanathan, K., Ahuja, K.K., and Panda, J., "The Sources of Jet Noise: Experimental Evidence," $13^{\text {th }}$ AIAA/CAES Aeroacoustics Conference, Rome, Italy, May 2007, AIAA 2007-3641.

[10] Simonich, J.C., Narayanan, S. and Schlinker, R.H., "Facility and Data Quality Issues for Scale-Model Jet Noise Testing," $41^{s t}$ Aerospace Sciences Meeting, Reno, NV, January 2003, AIAA 2003-1057.

[11] Dougherty, R. P., "Beamforming in Acoustic Testing," in Aeroacoustic Measurements, edited by T. J. Mueller (Springer-Verlag, Berlin, 2002).

[12] Shannon, D., and Morris, S. C., "Trailing Edge Noise Measurements Using a Large Aperture Phased Array," International Journal of Acoustics, Vol. 7(2), pp. 147-176 (2008).

[13] Dougherty, R. P., "Extensions of DAMAS and Benefits and Limitations of Deconvolution in Beamforming." 11th AIAA/CEAS Aeroacoustics Conference, May 2005, Monterey, California, AIAA 2005-2961.

[14] Tanna, H.K., Dean P. D., Burrin, R. H., "The Generation and Radiation of Supersonic Jet Noise, Part III, Turbulent Mixing Noise Data," AFAPL-TR-76-65, 1976.

[15] Tanna, H.K, Dean, P.D, Fisher, M.J, "The Influence of Temperature on Shock-Free Supersonic Jet Noise", J. Sound Vib., Vol. 39(4), 1975, pp. 429-460. 
[16] Brown, C., Bridges, J., "Small Hot Jet Acoustic Rig Validation,” NASA TM -2006-213234.

[17] Bass, H.E., Sutherland, L.C., Zuckerman, A.J., Blackstock, D.T. and D.M. Hester, "Atmospheric Absorption of Sound: Further Developments," J. Acoustic Soc. Am., Vol. 97, Part 1, pp. 680-683, January 1995 and erratum J. Acoustic. Soc. Am., Vol. 99, Part 2, p. 1259, February 1996.

[18] Schlinker, R.H., Liljenberg, S.A., Polak, D.R., Post, K.A., Chipman, C. T., and Stern, A.M., "Supersonic Jet Noise Source Characteristics and Propagation: Engine and Model Scale,", $14^{\text {th }}$ AIAA/CEAS Aeroacoustics Conference, Rome, Italy, May 2007, AIAA 2007-3623. 